\title{
Instructional Strategies to Manage Cognitive Load in Orthodontics
}

\author{
Saad Asad ${ }^{1}$
}

\begin{abstract}
Introduction: Medical Education has recently taken its position in Health professional education, however still it is not very popular in Dentistry, though professional are working hard to apply its principals. In the recent past Orthodontic education has been revolutionized and instructional strategies and designs to reduce the cognitive load and improve learner's germane load have been employed. However literature discusses educationist`s perspective while student's perspective regarding this have not been explored much. Aim of this study is to assess student's perspective about different instructional strategies discussed through examples used to manage cognitive load in Orthodontic Education.
\end{abstract}

Materials and Method: The study was conducted through a 14 instrument questionnaire on a sample consisted of Sixty Six final year BDS students (12 males and 44 females) from University College of Dentistry, The University of Lahore.

Results: Student's perspective through orthodontic examples for instructional strategies to manage cognitive load was assessed. Dental students reported positively to all questions in the questionnaire except the question regarding Goal free principle.

Conflict of Interest: No

Funding Source: No

Asad S. ${ }^{1}$

Associate Professor, Head Department of Orthodontics and Oral Biology and Tooth Morphhology, University College of Dentistry, The University of Lahore
Results: Different instructional strategies to reduce extrinsic load, manage intrinsic load and optimize germane load through examples from orthodontic syllabi have been given.

Conclusion: Student's perspective as assessed through questionnaire is mostly in accordance with the literature, except for goal free principle where only $25.75 \%$ student responded that this instructional strategy will improve learning.

Key Words: Cognitive Load, Instructional Strategies, Orthodontic / Dental Education.

\section{Introduction}

It is important for teachers to find the "sweet spot" for engaging all students in learning. Orthodontic education in the same context has been revolutionized. Rather than didactic lectures there is a blend of interactive lectures, small group teaching, demonstrations and work based teaching. All of these strategies mend to reduce the cognitive load of the subject and tend to improve the student learning. Managing cognitive load for appropriate student learning is thus pivotal in an educational setup; hence it is important to understand applications of Cognitive Load Theory (CLT) in medical education in general and in orthodontics specially. ${ }^{1}$

Cognitive load theory (CLT) was presented by John Sweller in 1988 that relates three key components of the human mind framework as shown in Fig 1. ${ }^{2-4}$ It has three main types, Intrinsic Load, Extrinsic Load and Germane Load and by managing different loads learning can be improved. ${ }^{5}$

Intrinsic Load: Intrinsic Load is the core of the given task and is imposed by the elements in the content and interactivities. Tasks can range from low element interactivity to high element interactivity for example, 
Can hold $7 \pm 2$ information elements and process 2-4 elements at time

Visual Memory:

Sensory

Memory

less than 0.25

seconds

Large Capacity
Rehearsal

Working

Memory

30 seconds
Long Term Memory

holds knowledge for long period of time

Auditory Memory:

Visual memory and auditory memory are independent and working memory capacity can be improved by utilizing both.

Fig. 1: Inter-relation between sensory, working and long term memory.

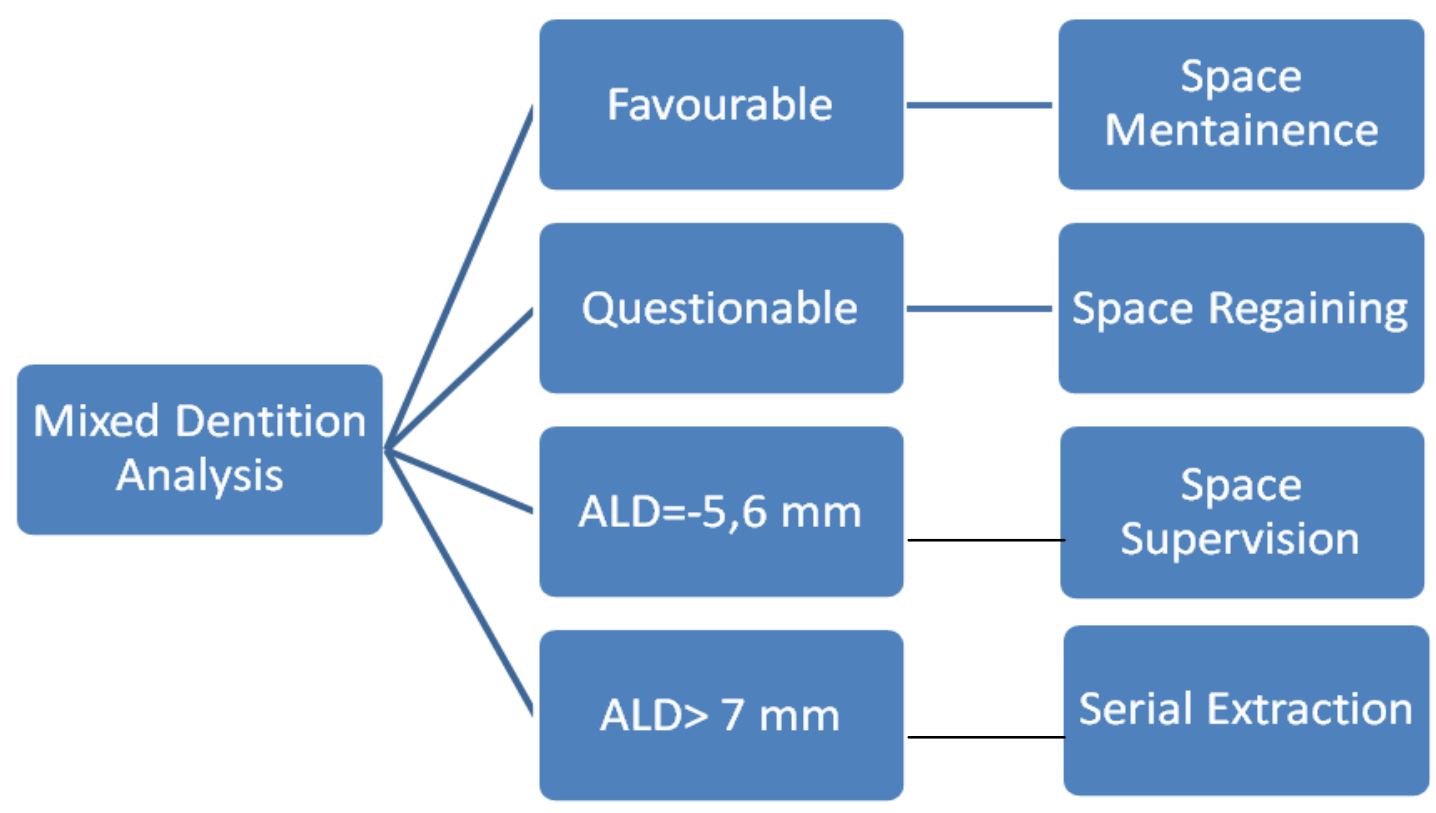

Fig. 2: Schema formation to reduce the cognitive load in high interactivity situations.

when Mixed Dentition Analysis is taught in isolation, then this will be an example of low element interactivity, however if we teach them Mixed dentition Analysis with its clinical relevance in Space Manage- ment Protocol then this will be an example of high interactivity and will impose high cognitive load on a novice learner. Schema construction however can change high element interactivity to simplified form there- 
by reducing the intrinsic load as shown in fig 2 . Schema when gets automated they become an innate behavior. Most of the recent books in medical teaching uses the concept of schema for better learning. ${ }^{1,2,5-7}$

Extrinsic Load: Extrinsic load is unwanted component associated with the given task and is unconsciously imposed by poorly designed instructional materials. It can be minimized by avoiding distracters and reducing complexities. For example, In Orthodontics if on the very $1^{\text {st }}$ day of clinical setting teacher ask students to place brackets on patients teeth without demonstrating them, then this will be an example of extrinsic load. That extrinsic load however can be managed if teacher provides an example that how he / she places the brackets first before asking students to do so on their own. ${ }^{1,2,5-7}$

Germane Load: Germane load is processing that facilitates effective learning and is enhanced by better instructional strategies such as problem based learning or case based learning. ${ }^{1,25-8}$ For example if teacher has to discuss classification of malocclusion in relevance with the diagnosis and treatment planning then intrinsic load of this topic is high. Extrinsic load of this topic has to be reduced by utilizing different instructional designs, which will improve the germane load.

Various instructional strategies have been proposed in the literature to manage different types of cognitive load thereby improving the overall learning. However literature discusses educationist's perspective while student's perspective regarding this have not been explored much. Aim of this study is to assess student's perspective about different instructional strategies discussed through examples used to manage cognitive load in Orthodontic Education.

\section{Material and Methods}

The study sample consisted of Sixty Six final year BDS students (12 males and 44 females) from University College of Dentistry, The University of Lahore. After obtaining informed consent they were given a questionnaire. Questionnaire comprised of 14 instruments (07 questions to reduce extrinsic load, 03 questions to manage intrinsic load and 04 question to optimize germane load) each testing one instructional strategy used in literature to manage cognitive load as given in fig 3. Since knowing the name of exact instructional strategy is beyond their scope so examples related to orthodontics were carved out that relates to the instructional strategy the best. Data was then analyzed using SPSS 17.0 and frequency and percentage for each instrument for each subject was then calculated.

\section{Results}

Student's perspective regarding orthodontic examples for instructional strategies to manage cognitive load has been discussed in table 1 .

\section{Discussion}

Various instructional strategies use to decrease extrinsic load, manage intrinsic load and optimize germane load proposed by Sweller J and John Q, have been tested through a questionnaire and student's perspective has been evaluated. ${ }^{1,6}$

\section{Reducing Extrinsic Load}

08 questions were used to assess the instructional strategies used to reduce extrinsic load.

\section{Goal-Free Principle. ${ }^{1,6,9,10}$}

Goal Free Principle suggests replacing conventional tasks with goal-free tasks. For example while teaching Treatment Planning in Orthodontics rather than asking students to make most appropriate treatment plan for the given case, it would be better to ask them to give possible treatment options for the case in question. This will help them to explore in better way, develop schemas and understand things effectively. When this instructional strategy was questioned in this study through an example only $25.75 \%$ student responded that this will improve learning. This suggests that undergraduate students are keener to be given readymade single treatment option thereby learning through memorizing strategies rather than inculcating into thinking process. Interestingly however position holders and who have O-level / A-level background were among those who responded that this strategy is better for learning. $1,6,9,10$

\section{Worked Example ex,-12 $^{1,9}$}

Worked Example provides learner with at least one demonstration before asking the learners to search for the solution themselves. For Example let students criticize a ready-made treatment plan several times for 
Table 1: Frequency and Percentage of each question used to assess the instructional strategies to manage cognitive load and thus better learning.

\begin{tabular}{|c|c|c|c|c|c|c|c|c|c|c|c|c|c|c|}
\hline & Q1 & & Q2 & & Q3 & & Q4 & & Q5 & & Q6 & & Q7 & \\
\hline & $\mathrm{n}$ & $\%$ age & $\mathrm{n}$ & $\%$ age & $\mathrm{n}$ & \%age & $\mathrm{n}$ & $\%$ age & $\mathrm{n}$ & \%age & $\mathrm{n}$ & $\%$ age & $\mathrm{n}$ & $\%$ age \\
\hline Agree & 32 & 48.48 & 28 & 42.42 & 28 & 42.42 & 38 & 57.57 & 30 & 45.45 & 21 & 31.81 & 31 & 22.72 \\
\hline Neutral & 17 & 25.75 & 17 & 25.75 & 22 & 33.33 & 12 & 18.18 & 22 & 33.33 & 15 & 22.72 & 20 & 30.30 \\
\hline \multirow[t]{2}{*}{ Disagree } & 17 & 25.75 & 21 & 31.81 & 16 & 24.24 & 16 & 24.24 & 14 & 21.21 & 30 & 45.45 & 15 & 46.96 \\
\hline & 66 & 100 & 66 & 100 & 66 & 100 & 66 & 100 & 66 & 100 & 66 & 100 & 66 & 100 \\
\hline & & & & & & & & & & & & & & \\
\hline \multirow[b]{3}{*}{ Agree } & Q8 & & Q9 & & Q10 & & Q11 & & Q12 & & Q13 & & Q14 & \\
\hline & $\mathrm{n}$ & $\%$ age & $\mathrm{n}$ & $\%$ age & $\mathrm{n}$ & \%age & $\mathrm{n}$ & $\%$ age & $\mathrm{n}$ & $\%$ age & $\mathrm{n}$ & \%age & $\mathrm{n}$ & $\%$ age \\
\hline & 28 & 42.42 & 39 & 59.09 & 24 & 36.36 & 30 & 45.45 & 35 & 46.96 & 44 & 66.66 & 46 & 69.69 \\
\hline Neutral & 20 & 30.30 & 13 & 19.69 & 24 & 36.36 & 22 & 33.33 & 24 & 36.36 & 17 & 25.75 & 12 & 18.18 \\
\hline \multirow[t]{2}{*}{ Disagree } & 18 & 27.27 & 14 & 21.21 & 18 & 27.27 & 14 & 21.21 & 7 & 10.60 & 5 & 7.57 & 8 & 12.12 \\
\hline & 66 & 100 & 66 & 100 & 66 & 100 & 66 & 100 & 66 & 100 & 66 & 100 & 66 & 100 \\
\hline
\end{tabular}

different types of orthodontic cases before having them independently generate treatment plan for their cases. Critically evaluating the developed treatment plan for say different Class II cases before making a treatment plan themselves will help them know why a typical treatment plan is selected for a given case, what are its advantages and limitations over other treatment options. Another Example for adopting this principle for managing extrinsic load is that teacher should demonstrate ALD assessment themselves before actually asking students to perform and analyze. $42.42 \%$ of the students responded positive to this question which is in agreement with what is suggested by the literature. , $^{1,9-12}$

\section{Problem Completion ${ }^{1,6,9-12}$}

Problem completion provide learner with a partially completed problem and ask them to complete the missing steps. For Example when we have to teach Composite Cephalometric Analysis to students ask them to do Sagital Skeletal Analysis first, then Vertical analysis, then the dental analysis and finally the soft tissue analysis rather than asking them to do the whole Cephalometric analysis in one go. Another Example is that if you are given partial problems first and then subsequently given the whole case to plan will impose less cognitive load then if you are given a cases to solve in one go. This step wise sequential building of know- ledge builds on learning. $42.42 \%$ of the students responded positive to first question while $57.57 \%$ responded positive to second question which supports role of this instructional strategy in reducing extrinsic load. ${ }^{1,6,9-12}$

\section{The Signaling Effect ${ }^{4}$}

The Signaling Effect suggests that people learn better when the material is organized with clear outlines and headings. When teachers are teaching orthodontics, material should be organized For Example when orthodontic diagnosis is taught in the classes at the same time they should be discussed and hands on activities should be performed in the clinics. $45.45 \%$ of the students responded positive to this question 5 whi$\mathrm{ch}$ is in agreement with what is suggested by the literature. $^{4}$

\section{Split Attention ${ }^{1,6,10-15}$}

Split Attention means replace multiple sources of information, distributed either in space or time, with one integrated source of information For Example when making an academic schedule interactive lecturing of each topic must be in line with the clinical assignment, such as teaching impression technique for Cleft lip and Palate must be followed by a clinical demonstration and Hands on application. Similarly 
when teaching Classification of Dentofacial Deformity it must be followed by application of the same on patients with different malocclusion. Integrating thus will facilitate learning better. Question 5 also tested the Split attention principle and found that $45.45 \%$ of the students responded positive to this. ${ }^{1,6,10-15}$

\section{Modality Principle 1,6,9-12 $^{1,10}$}

Modality principle refers to presenting information in both visual and auditory modalities. For Example when you are delivering an interactive lecture on the parts of functional appliance such as Twin Block, manage the cognitive load by showing students the appliance in small groups as well. Sowing them how to make the appliance and then asking them to make the appliance them self will further aid in understanding of the concepts. Learning can be further improved if the components are related with their function by showing them a video. $45.45 \%$ of the students responded that modality principle is effective in reducing cognitive load and improving learning. ${ }^{1,6,9-12}$

\section{Redundancy Principle (1,6-12 $^{1,-19}$}

Redundancy Principle means replacing multiple sources of information that are self - contained with one source of information. For Example better learning takes place when the topic is discussed by one of the most effective way of explaining it rather discussing same topic in both written format and through a flow chart. Only $22.72 \%$ of the students responded positive to the question related to redundancy principle which actually means that student still wants that things should be repeatedly explained and in different formats. ${ }^{1,69-12}$

\section{Personalization Principle ${ }^{4,8}$}

Personalization Principle suggests that people learn better when words presented are conversational rather than expository. For Example when teaching orthodontics, conversational mode of teaching should be adopted to gain the interest. Etiology of Cleft Lip and Palate rather than discussing flat can be placed in an interactive way such as:

"Parents of new born baby with a unilateral cleft lip come to the neonatologist. Parents were concerned about why this happened to their baby. Mother of the baby was herself having the repaired cleft of the lip. What could be the most possible cause of Cleft Lip and palate in this case?"
$42.42 \%$ of the students responded to this question positively which is in agreement with literature supporting Personalization Principle. ${ }^{4,8}$

Thus extrinsic load can be reduced employing above mentioned principles, this will reduced cognitive load and will induce conceptual, analytical and inquisitive approach in students.

\section{Managing Intrinsic Load}

Intrinsic Load can't be reduced by instructional designs but can be managed through different instructional strategies in Orthodontics education.

\section{Simple-to-complex Strategy ${ }^{1,6,9,10,16,17}$}

Gradually replace tasks with relatively low complexity with tasks having full complexity. For Example we can ask our students to place one/ two bracket first on the patients and then few brackets and finally we can ask them to bond both arches. Similarly we can ask our students to replace few ligatures first and then gradually we can bring them to the level that they can change the ligatures in both arches. $59.09 \%$ of the students responded positively to question related to simple to complex strategy. $1,6,9,10,16,17$

\section{Progress from low-to high-physical fidelity ${ }^{1,6,9,10,16,17}$}

Work from tasks with a low physical fidelity to tasks with a higher physical fidelity. For Example we can manage intrinsic load by discussing paper-based patient cases at the commencement of the program to scenarios using mannequins and then to the real patients. $59.09 \%$ of the students responded positively to the question related to this principle which is in accordance to what is reported in literature. , $^{1,6,9,10,16,17}$

\section{Two-phase isolated-interactivity strategy ${ }^{7}$}

People learn better through two-phase isolated-interactivity strategy, whereby isolated elements are presented in the first phase so that learner can form schema, while in the $2^{\text {nd }}$ phase explanations and interactions of different elements are explained. ${ }^{7}$ For Example while teaching Cephalometric analysis first demonstrates land marks, than lines and angles, then actual analysis and finally interpretations. $45.45 \%$ of the students responded positively to the question related to this principle which is in accordance to what is reported in literature. ${ }^{7}$ 


\section{Optimizing Germane Load}

Aim of teaching is to ultimately improve the Germane Load by reducing extrinsic load and managing intrinsic load through different instructional designs.

\section{Contextual Interference ${ }^{1,6,9,18,19}$}

Different versions of a task are practiced in a random rather than blocked order. For Example in perspective of Growth \& Development if we have to discuss the derivatives of Pharyngeal arches rather than asking them to list the derivatives of each arch, we can ask them to specify that from which pharyngeal arch Ear Osssicles gets derived, from which pharyngeal arches tongue gets derived etc. This will improve the Germane Load i.e. the learning. 53.03\% of the students responded positively to question no. 13 related to this principle which is in accordance to what is reported in literature. ${ }^{1,6,9,18,19}$

\section{Variability ${ }^{1,6,9,18,19}$}

Learning is enhanced when the variability of the task/ problem is increased. For Example when discussing management of Thumb Sucking we can discuss its management in different age groups or we can discuss it under the headings of Preventive, Interceptive, comprehensive and surgical Orthodontics. This variability and comparison improves the learning process. $53.03 \%$ of the students responded positively to question no 13 related to this principle which is in accordance to what is reported in literature. ${ }^{1,6,9,18,19}$

\section{Self-Explanation Principle Pr,6,9,18,19 $^{10}$}

Replace separate worked examples or completion tasks with enriched ones containing prompts, asking learners to self-explain the given information. For Example for students learning Orthodontic Biomechanics, present an animation of say $\mathrm{V}$ Bend works and provide prompts that ask them to self - explain the underlying mechanisms. This will improve the think process and thus the learning. $66.67 \%$ and $69.69 \%$ of the students responded positively to question no 14 and 15 respectively related to this principle which is in accordance to what is reported in literature. ${ }^{1,6,9,18,19}$

\section{Imagination ${ }^{1,6}$}

Learners perform better when asked to imagine a concept or procedure rather than by studying it. For Example Ask the students to visualize the steps of the procedure such as TAD placement before actually applying it on the patient. $66.67 \%$ and $69.69 \%$ of the students responded positively to question no 14 and 15 respectively related to this principle which is in accordance to what is reported in literature. , $^{1,6,18,19}$

\section{Conclusion}

While teaching Orthodontics we can mange cognitive load of the subject by using different instructional strategies. So it is important for course designers, curriculum designers and teachers to utilize these strategies to make the subject more understanding thereby enhanceing the learning process of the students. Student's perspective in this regards as assessed through questionnaire is mostly in accordance with the literature, except for goal free principle where only $25.75 \%$ student responded that this instructional strategy will improve learning.

\section{References}

1. Van Merriënboer JJG, Sweller J. Cognitive load theory in health professional education: Design principles and strategies. Med Educ. 2010; 44 (1): 85-93.

2. Qiao YQ, Shen J, Liang X, Ding S, Chen FY, Shao L, et al. Using cognitive theory to facilitate medical education. BMC Med Educ. 2014 Jan; 14: 79.

3. Jong T. Cognitive load theory, educational research, and instructional design: some food for thought. Instr Sci. 2009; 38 (2): 105-34.

4. Mayer RE. Applying the science of learning to medical education. Med Educ. 2010; 44 (6): 543-9.

5. Khalil MK, Paas F, Johnson TE, Payer AF. Interactive and dynamic visualizations in teaching and learning of anatomy: A cognitive load perspective. Anat Rec - Part B New Anat. 2005; 286 (1): 8-14.

6. Young JQ, Van Merrienboer J, Durning S, Ten Cate O. Cognitive Load Theory: implications for medical education: AMEE Guide No. 86. Med Teach. 2014; 36 (5): 371-84.

7. Sweller J, Ayres P, Kalyuga S. Intrinsic and Extraneous Cognitive Load. Cognitive Load Theory, 2011: p. 5769.

8. Crowell A, Schmeichel BJ. Approach motivation and cognitive resources combine to influence memory for positive emotional stimuli. Cogn Emot. 2015 Feb. 3: 19.

9. Qiao YQ, et al. Using cognitive theory to facilitate medical education. BMC Med Educ. 2014 Apr 14; 14: 79.

10. Leppink J, van den Heuvel A. The evolution of cognitive load theory and its application to medical education. Perspect Med Educ. 2015 Jun; 4 (3): 119-27. 
11. Haji FA et al. Measuring cognitive load during simulation - based psychomotor skills training: sensitivity of secondary - task performance and subjective ratings. Adv Health Sci Educ Theory Pract. 2015 Mar 12.

12. Sweller J, Cooper M. The use of worked examples as a substitute for problem solving in learning algebra. Cogn Instr. 1985; 1: 59-89.

13. Chandler P, Sweller J. Cognitive load theory and the format of instruction. Cogn Instr. 1991; 8: 293-332.

14. Chandler P, Sweller J. The split - attention effect as a factor in the design of instruction. Brit J Educ Psych. 1992; 62: 233-46.

15. Cierniak G, Scheiter K, Gerjets P. Explaining the split attention effect: is the reduction of extraneous cognitive load accompanied by an increase in germane cognitive load? Comp Human Behav. 2009; 25: 315-24.

16. Sweller J. Element interactivity and intrinsic, extraneous, and germane cognitive load. Educ Psych Rev. 2010; 22: 122-38.

17. Ayres P. Using subjective measures to detect variations of intrinsic load within problems. Learn and Instr. 2006; 16: 389-400.

18. Debue N, van de Leemput C. What does germane load mean? An empirical contribution to the cognitive load theory. Front Psychol. 2014 Oct 1; 5: 1099.

19. DeLeeuw K. E., Mayer R. E. A comparison of three measures of cognitive load: evidence for separable measures of intrinsic, extraneous, and germane load. J. Educ. Psychol. 2008; 100: 223-234 10. 